\title{
Discours médiatique spécialisé : la vulgarisation (popularisation) à la BBC Radio
}

\section{Danielle Clément-Guiraud}

\section{(2) OpenEdition}

1 Journals

Édition électronique

URL : http://journals.openedition.org/asp/645

DOI : $10.4000 /$ asp. 645

ISBN : 978-2-8218-0402-9

ISSN : 2108-6354

\section{Éditeur}

Groupe d'étude et de recherche en anglais de spécialité

Édition imprimée

Date de publication : 1 décembre 2006

Pagination : 49-61

ISSN : 1246-8185

\section{Référence électronique}

Danielle Clément-Guiraud, « Discours médiatique spécialisé : la vulgarisation (popularisation) à la BBC Radio », ASp [En ligne], 49-50 | 2006, mis en ligne le 05 février 2010, consulté le 22 mars 2021. URL : http://journals.openedition.org/asp/645; DOI : https://doi.org/10.4000/asp.645

Ce document a été généré automatiquement le 22 mars 2021.

Tous droits réservés 


\title{
Discours médiatique spécialisé : la vulgarisation (popularisation) à la BBC Radio
}

\author{
Danielle Clément-Guiraud
}

\section{Introduction}

1 L'étude des discours des milieux professionnels a beaucoup moins exploré les productions langagières transmises par le canal oral. Il peut s'agir d'un choix théorique. Lerat (1995 : 12) estime en effet que, si la pratique est liée à l'oralité, l'écrit est un vecteur obligé des connaissances et il exclut l'oral du champ de son étude car « la première spécificité des langues spécialisées est qu'elles relèvent de l'écrit ». Toutefois, avec le développement de la pragmatique et de l'analyse conversationnelle ${ }^{1}$, les chercheurs se sont intéressés aux discours dans leur interaction en milieu professionnel (voir Drew et Heritage 1992) et l'analyse de la langue parlée au travail peut avoir des applications pratiques permettant d'améliorer son efficacité comme véhicule d'information et de communication ${ }^{2}$.

2 Pour expliquer le petit nombre d'études portant sur la langue parlée des milieux professionnels, il convient de prendre en compte la difficulté de collecter et d'étudier ces énoncés. Or, nous postulons que la BBC Radio a élaboré un genre qui constitue une voie d'accès relativement simple à la culture et aux discours de certaines professions : ces magazines, diffusés sur des stations grand public que la $B B C$ qualifie de quality speech services (Radio 4 et BBC World Service), ont pour objectif de transmettre des connaissances et de communiquer des informations dans des domaines spécialisés (juridique, scientifique, médical, économique, etc.). Certes, il s'agit alors de discours seconds $^{3}$, discours de professionnels sur leur pratique et sur leur domaine de compétence dans le cadre d'une communication de masse. De plus, dans le continuum des discours parlés, le discours radiophonique ou télévisuel se rapproche du discours écrit par certaines caractéristiques (le différé du message, la virtualité du récepteur, 
l'éloignement ou l'absence de réponse, l'impossibilité d'un échange immédiat) et par le fait qu'il peut être enregistré, stocké et faire l'objet de différents traitements (voir Moirand 1983, Maingueneau 2002). Il nous semble néanmoins que ces magazines radiophoniques permettent d'entendre un discours de popularisation qui peut intéresser légitimement à la fois les spécialistes du domaine médiatique et les spécialistes du domaine d'application du secteur LANSAD (langues pour spécialistes d'autres disciplines).

3 Dans un premier temps, nous évoquerons les conditions historiques et le contexte social qui, tout en façonnant le service public de la BBC, ont rendu possibles la naissance et le développement de ce genre voué à la popularisation de connaissances scientifiques et techniques. Nous présenterons ensuite le cadre médiatique dans lequel ces magazines spécialisés se situent de nos jours. Enfin, un rapprochement entre deux études réalisées dans le cadre du GERAS (sur une émission scientifique et sur une émission juridique) mettra en évidence des caractéristiques communes à ces magazines.

\section{Les origines de la BBC et les concepts fondateurs}

4 L'histoire de cette prestigieuse institution britannique commença en 1922 par la création de la British Broadcasting Company ${ }^{4}$, consortium de droit privé qui regroupait les entreprises concernées afin d'organiser la radiodiffusion sur les ondes de manière rationnelle. Rien ne prédisposait cette structure commerciale à devenir un service public et cette évolution est essentiellement due à la volonté de John Reith, qui dirigea ce consortium en tant que managing director et qui continua à assumer ces fonctions en tant que director-general quand la BBC devint, en 1927, une corporation, c'est-à-dire une société de droit public. Homme de principes moraux et religieux stricts, il continua à diriger la BBC d'une main de fer jusqu'en 1936. Ses convictions concernant le rôle éducatif et culturel de la radio, partagées par la plupart de ses collaborateurs, fondèrent le service public britannique et influencèrent de nombreux autres pays. Le site de la $\mathrm{BBC}^{5}$ les résume ainsi : «Reith's vision was of an independent British broadcaster able to educate ${ }^{6}$, inform and entertain the whole nation, free from political interference and commercial pressure ».

\subsection{Une mission éducative et culturelle et un souci démocratique}

Soustraire la $\mathrm{BBC}$ aux pressions économiques et politiques était une condition essentielle pour un service public national. En 1924, alors que la BBC était toujours un consortium d'intérêts privés, Reith écrivait :

I think it will be admitted by all that to have exploited so great a scientific invention for the purpose and pursuit of entertainment alone would have been a prostitution of its powers and an insult to the character and intelligence of the people ${ }^{7}$.

Reith mit donc la fonction éducative au centre de sa conception du service public et, à une époque où la scolarité de la plupart des adultes s'arrêtait à quatorze ans, il prit l'initiative d'associer la BBC aux services d'enseignement des adultes. La Charte Royale qui constitua la BBC énonce les mêmes priorités dans ses objectifs : educate, inform and entertain. 
7 La radio devait aussi concourir à élever la moralité et le bon goût populaire : les présentateurs, qui sortaient des meilleures universités, s'exprimaient avec l'accent de la classe cultivée, ou received pronunciation ${ }^{8}$; même les vêtements qu'ils portaient pour parler devant le microphone devaient refléter l'importance de leur mission. Si les contraintes imposées par la corporation se sont assouplies, les journalistes reçoivent encore le Producer's Guidelines ${ }^{9}$, code de déontologie très détaillé, qui permet de veiller au respect des obligations de la $\mathrm{BBC}$, de protéger les auditeurs et les téléspectateurs de toute exploitation. Le sens civique est donc un élément constitutif de l'« homo sapiens $\mathrm{BBC}^{10}$ ", au même titre que la compétence technique et la rigueur scientifique.

8 Selon Cusin Berche (2002: 603), une activité vulgarisatrice ne peut se réaliser que « dans une société caractérisée par la présence d'un niveau scientifique élevé et animée par un souci démocratique ». La BBC réunissait donc ces deux caractéristiques qui favorisèrent la naissance d'un genre voué à la popularisation. L'existence de ces conditions nécessaires ne suffit cependant pas à expliquer l'efficacité communicationnelle de ces magazines ni le développement de formes journalistiques particulières qui caractérisent ces magazines spécialisés. Il convient d'envisager ici le rôle qu'a joué un mode de programmation particulier qui a allié une organisation stricte à une grande stabilité.

\subsection{Une programmation variée : le concept de mixed programming}

9 Reith entendait élever le niveau culturel et intellectuel de ses concitoyens par une programmation variée (mixed programming), qui attirerait de plus en plus d'auditeurs vers les programmes sérieux en alternant émissions éducatives ou culturelles et émissions de divertissement. Ce concept est décrit en ces termes par Scannel et Cardiff dans leur ouvrage sur la BBC :

Mixed programming offered a wide and diverse range of materials over the course of each day and week. Typically it included news, drama, sport, religion, music (light to classical), variety or light entertainment. Not only did it cater for different social needs (education, information, entertainment), but for different sectional interests within the listening public (children, women, businessmen, farmers, fishermen, etc.). (Scannell \& Cardiff cités par Crisell $1994: 21$ )

10 Cette dernière caractéristique, à savoir la volonté de réaliser, à côté des émissions d'intérêt général, des émissions qui s'adressent à des segments particuliers du public et à différentes catégories professionnelles, a aussi concouru à l'émergence d'émissions consacrées à des domaines et des milieux professionnels spécialisés.

11 De plus, un point de vue diachronique permet de constater une grande stabilité de programmation, qui a probablement permis l'élaboration progressive des traits spécifiques de ces magazines. Cette stabilité a été rendue possible par une longue situation de monopole, mais aussi par une fidélité aux principes fondateurs. Une comparaison entre les principes d'origineet ceux énoncés dans des rapports plus récents, par exemple le rapport Peacock ${ }^{11}$ en 1986 ou le rapport Thomas ${ }^{12}$ en 1999, met en avant une remarquable continuité dans la conception du service public audiovisuel.

12 La même fidélité se retrouve dans le mode de programmation et le choix des émissions. Ainsi, émissions culturelles ou d'information et feuilletons populaires alternent toujours à Radio 4, qui héberge des émissions cinquantenaires comme le feuilleton The Archers ou l'émission-débat Any Questions? ${ }^{13}$. Parmi les émissions spécialisées, le 
magazine économique et politique Analysis fut créé en 1970. Mais cette fidélité n'a pas sclérosé la $\mathrm{BBC}$ qui conjugue au contraire tradition avec innovation ${ }^{14}$ et a largement développé les nouveaux modes de communication comme Internet et la radio numérique ${ }^{15}$.

\section{Les magazines spécialisés à la BBC Radio}

\subsection{Les magazines spécialisés : des « news and current affairs » programmes}

Contrairement à la télévision publique dont l'audience subit une érosion régulière face à la concurrence des chaînes privées, la radio, qui avait semblé condamnée par l'avènement de la télévision et par la concurrence des stations commerciales, a su s'adapter ${ }^{16}$ sans renoncer aux grands principes du service public audiovisuel qu'elle a créé. Elle en tire aujourd'hui les profits puisque des études récentes montrent que, face aux stations commerciales et malgré leurs quatre-vingt ans, les stations radiophoniques de la «tante Beeb» attirent de plus en plus d'auditeurs ${ }^{17}$. Crisell (1984: 39) constate que le succès de Radio 4 , qui a conservé le mode de programmation traditionnel de la $\mathrm{BBC}$ et compte un nombre croissant d'auditeurs fidèles ${ }^{18}$, est en grande partie dû au journalisme d'information (news and current affairs).

Radio 4, partly drawing on its glorious past, eats its cake and has it: it offers mixed programming, yet manages to project a coherent identity as a spoken word/news and current affairs network. (Crisell $1984: 39$ )

L'expression news and current affairs semble parfois être l'association de deux concepts distincts : celui de l'information factuelle ${ }^{19}$ (news) et celui des current affairs, émissions qui complètent les bulletins d'information qu'elles analysent et éclairent, mais aussi magazines spécialisés comme Law in Action ou Science in Action, qui entretiennent avec l'actualité un lien bien moins étroit. D'autres usages de news and current affairs semblent se référer à un concept unique, celui d'information. Or, plus que l'aspect factuel de l'actualité, c'est la visée popularisatrice que l'un des réalisateurs de Science in Action, Chris Westcott, interviewé par Pierre Vuillaume, met en avant dans ce concept.

The important point about Science in Action as a programme is that it is a science news and current affairs programme, and should be accessible to everybody as any other kind of news and current affairs programme would be. (Vuillaume 1994 : 235)

\subsection{Une organisation modulaire}

L'observation de la grille des programmes de Radio 4 permet de constater que l'aprèsmidi (à partir de $12 \mathrm{~h}$ ) et la soirée (à partir de $18 \mathrm{~h}$ ) sont organisées de manière modulaire, le plus souvent en séquences de trente minutes où les émissions distrayantes alternent avec les émissions informatives, conformément aux principes du mixed programming. Parmi les émissions régulières, les magazines spécialisés (series) consacrés aux sciences, à la technologie, à la santé, au droit, à l'économie, au travail, aux mathématiques, à la finance, etc. sont programmés dans ces plages horaires ${ }^{20}$ et dans ces modules de trente minutes ${ }^{21}$.

Il existe par ailleurs des émissions régulières consacrées à des domaines professionnels, mais qui présentent des situations énonciatives différentes et qui n'ont pas pour 
objectif essentiel de populariser des connaissances. Les émissions interactives où les professionnels invités répondent aux questions des auditeurs (live phone-ins) ont une visée pratique, consumériste ${ }^{22}$. Les débats entre spécialistes du domaine (discussions) favorisent l'échange de points de vue plus que la transmission du savoir ${ }^{23}$.

Il faut aussi signaler l'existence d'émissions qui répondent aux mêmes objectifs et aux mêmes règles journalistiques et discursives que Law in Action et Science in Action mais qui ne présentent pas le même caractère de régularitée ${ }^{24}$. Il s'agit de séries limitées à deux, trois ou six émissions, parfois reprises à intervalles réguliers ${ }^{25}$.

Radio 4 propose donc toute une gamme de magazines dans les domaines des sciences dites dures comme dans le domaine des sciences humaines. C'est aussi le cas de BBC World Service qui a un financement, des obligations, un public et un mode de programmation différents, mais qui propose des magazines spécialisés de format et de facture identiques à ceux de Radio 4. Certains journalistes spécialisés collaborent aux deux stations, qui semblent partager la même culture radiophonique. Ainsi, Quentin Cooper, qui présente l'émission scientifique The Material World et technologique Connect ("regular technology-in-action documentary») sur Radio 4 collabore aussi à Science in Action sur BBC World Service. (< http://www.bbc.co.uk/radio4/presenters/ cooperq_biog.shtml>)

19 La grille des programmes témoigne du rapprochement entre les deux stations puisque, pendant la nuit, de $1 \mathrm{~h}$ à $5 \mathrm{~h} 30$, Radio 4 diffuse « a selection of news and current affairs, arts and science programmes ». Il s'agit bien des magazines spécialisés dont Pierre Vuillaume (1997: 647) note qu'ils représentent une source précieuse de documents pour l'enseignant d'anglais de spécialité.

20 Après avoir brièvement situé ces magazines dans la $\mathrm{BBC}$ Radio, un rapprochement entre certaines caractéristiques de Science in Action et de Law in Action mises en avant respectivement par Pierre Vuillaume (1993, 1994 et 1997) et Danielle Clément-Guiraud (2002) permettra de mieux appréhender ce genre radiophonique.

\section{Law in Action et Science in Action}

\subsection{La structure générale et le contenu thématique}

21 La structure générale de ces deux magazines est identique. Chaque émission dure un peu moins de 30 minutes et comporte trois phases: l'amorce, les sujets et la clôture. Dans l'amorce, le présentateur introduit les sujets de manière simple et attractive, dans un langage peu technique, parfois avec des éléments sonores ou musicaux. Le nombre de sujets varie, en général quatre ou cinq pour Law in Action, quatre en moyenne pour Science in Action.

Lest marqué dans les deux cas par la diversité et la technicité des sujets à mi-chemin entre un ancrage dans l'actualité et une approche de type encyclopédique. Pierre Vuillaume (1994:235) remarque que la richesse thématique des sujets de Science in Action lui avait initialement permis de sélectionner des extraits de son corpus pour un travail didactique avec des spécialistes en informatique et robotique ${ }^{26}$ et ensuite d'en sélectionner d'autres pour des étudiants de chimie. Et il en aurait été de même, écrit-il, pour la médecine, l'astronomie, la paléontologie, etc. La même diversité se retrouve dans les catégories juridiques, les lois, les affaires et les 
juridictions mentionnées dans quinze émissions de Law in Action (Clément-Guiraud $2002: 28-37)$.

\subsection{Le contrat de communication médiatique} Vuillaume (1994 : 232) comme double. «Un des principaux soucis des réalisateurs de cette émission est de ne pas oublier qu'ils s'adressent à [...] deux types d'auditeurs » : le profane, mais aussi le scientifique qui peut s'intéresser à un sujet hors de sa discipline, tel le professeur Jean-Claude Roman, Doyen de la Faculté d'Aix-Marseille 3 et familier de Science in Action, qui a reconnu: “ j'ai appris des choses qui sont totalement en dehors de ma spécialité." (Vuillaume 1994 : 234). Un rapprochement peut être effectué avec les propos de Marcel Berlins, interviewé sur sa démarche vulgarisatrice à la radio, à la télévision et dans la presse.

In 1987, I started a legal magazine called Law Magazine which was an attempt to do something like Nature or New Scientist; to have a magazine that was on sale to the general public and bought by lawyers and the general public. (Clément-Guiraud Annexes 2002 : 112-114)

Cette citation éclaire aussi les conditions de finalité et le rapprochement avec Nature et New Scientist montre très clairement que l'objectif est celui de la vulgarisation de qualité, notion plus couramment associée au domaine scientifique que juridique. Dans les deux magazines se manifeste donc la même volonté de transmettre des connaissances et de populariser un savoir.

Dernier élément du contrat de communication, les conditions de disposition (Comment ?) relèvent d'une analyse des stratégies de communication de ce savoir par 
les journalistes. Nous avançons l'hypothèse qu'il s'agit de stratégies particulières liées à la visée vulgarisatrice (Clément-Guiraud 2002 : 48-88).

\section{Conclusion}

En reprenant les critères dégagés par Swales pour définir un genre (1990:58), on constate que ces deux magazines "exhibit various patterns of similarity in terms of structure, style, content, and intended audience ». Il est certain que deux est un nombre insuffisant pour constituer " a class of communicative events » (Swales 1990:46), mais il est vraisemblable que la plupart de ces caractéristiques communes se retrouvent dans les autres magazines spécialisés de Radio 4 et de BBC World Service. Si cette hypothèse était confirmée, il serait avéré que la volonté vulgarisatrice de la $B B C$ a créé un genre de popularisation des connaissances à la radio, produit d'une rencontre entre le savoir et la volonté démocratique.

Dans le cadre du secteur LANSAD, il nous semble que le caractère professionnel et la rigueur scientifique de ces magazines en font un objet pertinent d'étude et d'observation pour un travail sur les milieux professionnels et leurs discours. Par ailleurs, ces documents à visée popularisatrice ont des qualités pédagogiques qui permettent d'en faire un support oral d'enseignement attractif; en effet, en tant qu'objets de communication médiatique ancrés dans l'actualité et la civilisation britannique, ces émissions permettent de transmettre des connaissances scientifiques et techniques dans leur contexte culturel.

\section{BIBLIOGRAPHIE}

Berridge, Clive. 2000. « Bringing the law to the public: Clive Berridge interviews legal author and broadcaster Marcel Berlins ». BerridgeLaw consulté le 05/10/2000 <http:// www.lawbooks.freeserve.co.uk/marcel\%20berlins\%20interview/index.html>.

Charaudeau, Patrick. 1997. Le discours d'information médiatique. La construction du miroir social. Paris : Nathan.

Clément-Guiraud Danielle. 2002. « Formes du discours sur le droit anglais dans une émission de la BBC Radio 4 Law in Action ». Mémoire de DEA de langue anglaise des spécialités scientifiques et techniques, Universités de Bordeaux 2, Montpellier 3, Toulouse 1, Paris 4-Sorbonne, ENS Cachan.

Crisell, Andrew. 1994. Understanding Radio. Londres : Routledge.

Cusin-Berche, Fabienne. 2002. «Vulgarisation ». In Charaudeau, Patrick \& Dominique Maingueneau (dir.), Dictionnaire d'analyse du discours. Paris : Seuil, 603-605.

Drew, Paul \& John Heritage (dir.). 1992. Talk at Work: Interaction in Institutional Settings. Cambridge : Cambridge University Press.

Johnson, Edward. 2000. « Talking across Frontiers 1 ». Consulté le 18/02/2005 <http:// www.prolingua.co.uk/talking.pdf>. 
Lerat, Pierre. 1995. Les langues spécialisées. Paris : Presses Universitaires de France.

Maingueneau, Dominique. 2002. «Écrit/oral ». In Charaudeau, Patrick \& Dominique Maingueneau (dir.), Dictionnaire d'analyse du discours. Paris : Seuil, 202-205.

Moirand, Sophie. 1983. Situations d'écrit : compréhension, production en français langue étrangère.

Paris : CLÉ International.

Swales, John. 1990. Genre Analysis. English in Academic and Research Settings. Cambridge : Cambridge University Press.

Vuillaume, Pierre. 1993. «Science in Action (BBC World Service) : instrument de progrès en compréhension orale pour un groupe d'élèves-ingénieurs faux débutants? ». Mémoire de DEA de langue anglaise des spécialités scientifiques et techniques, Universités de Bordeaux 2, Montpellier 3, Toulouse 1, Paris 4-Sorbonne.

Vuillaume, Pierre. 1994. « 'From jelly babies to Roman candles'- Science et culture dans Science in Action (BBC World Service) ». ASp 5-6, 231-236.

Vuillaume, Pierre. 1997. « La vulgarisation scientifique sur les ondes internationales : BBC World Service - Radio France Internationale ». ASp 15-18, 647-657.

\section{ANNEXES}

Magazines spécialisés proposant des services en ligne (consultés en 2002)

\section{BBC Radio 4}

\begin{tabular}{|c|c|c|}
\hline Analysis & $\begin{array}{l}\text { <http://news.bbc.co.uk/2/hi/programmes/analysis/ } \\
\text { default.stm> } \\
\text { economics and political science }\end{array}$ & $\begin{array}{l}\text { Écoute } \\
\text { différée } \\
\text { Transcriptions }\end{array}$ \\
\hline Connect & $\begin{array}{l}\text { <http://www.bbc.co.uk/radio4/science/connect.shtml> } \\
\text { science and technology }\end{array}$ & $\begin{array}{l}\text { Écoute } \\
\text { différée }\end{array}$ \\
\hline $\begin{array}{l}\text { Farming Today } \\
\text { this week }\end{array}$ & $\begin{array}{l}\text { <http://www.bbc.co.uk/radio4/news/farmingtoday/ } \\
\text { index.shtml> } \\
\text { farming }\end{array}$ & $\begin{array}{l}\text { Écoute } \\
\text { différée }\end{array}$ \\
\hline $\begin{array}{l}\text { Food Programme, } \\
\text { the }\end{array}$ & $\begin{array}{l}\text { <http://www.bbc.co.uk/radio4/factual/ } \\
\text { foodprogramme.shtml> } \\
\text { food and farming }\end{array}$ & $\begin{array}{l}\text { Écoute } \\
\text { différée }\end{array}$ \\
\hline Frontiers & $\begin{array}{l}\text { <http://www.bbc.co.uk/radio4/science/frontiers.shtml> } \\
\text { science }\end{array}$ & $\begin{array}{l}\text { Écoute } \\
\text { différée }\end{array}$ \\
\hline In Business & $\begin{array}{l}\text { <http://www.bbc.co.uk/radio4/news/inbusiness.shtml> } \\
\text { business }\end{array}$ & $\begin{array}{l}\text { Écoute } \\
\text { différée }\end{array}$ \\
\hline
\end{tabular}




\begin{tabular}{|c|c|c|}
\hline Law in Action & $\begin{array}{l}\text { <http://www.bbc.co.uk/factsheets/lawinaction/> } \\
\text { law }\end{array}$ & $\begin{array}{l}\text { Écoute } \\
\text { différée } \\
\text { Factsheets }\end{array}$ \\
\hline Leading Edge & $\begin{array}{l}<\text { http://www.bbc.co.uk/radio4/science/leadingedge.shtml> } \\
\text { science }\end{array}$ & $\begin{array}{l}\text { Écoute } \\
\text { différée }\end{array}$ \\
\hline Living World & $\begin{array}{l}\text { <http://www.bbc.co.uk/radio4/science/livingworld.shtml> } \\
\text { science: natural history }\end{array}$ & $\begin{array}{l}\text { Écoute } \\
\text { différée }\end{array}$ \\
\hline Material World, the & $\begin{array}{l}\text { <http://www.bbc.co.uk/radio4/science/ } \\
\text { thematerialworld.shtml> } \\
\text { science }\end{array}$ & $\begin{array}{l}\text { Écoute } \\
\text { différée }\end{array}$ \\
\hline Money Box & $\begin{array}{l}\text { <http://news.bbc.co.uk/2/hi/programmes/moneybox/> } \\
\text { finance }\end{array}$ & $\begin{array}{l}\text { Écoute } \\
\text { différée } \\
\text { Transcriptions }\end{array}$ \\
\hline More or Less & $\begin{array}{l}\text { <http://news.bbc.co.uk/1/hi/programmes/more_or_less/> } \\
\text { mathematics (numbers and their place in the world) }\end{array}$ & $\begin{array}{l}\text { Écoute } \\
\text { différée }\end{array}$ \\
\hline Nature & $\begin{array}{l}\text { <http://www.bbc.co.uk/radio4/science/nature.shtml> } \\
\text { science: natural history }\end{array}$ & $\begin{array}{l}\text { Écoute } \\
\text { différée }\end{array}$ \\
\hline Nice Work & $\begin{array}{l}<\mathrm{http}: / / \text { www.bbc.co.uk/radio4/news/nicework.shtml> } \\
\text { working life (legal, psychological, economic...) }\end{array}$ & $\begin{array}{l}\text { Écoute } \\
\text { différée } \\
\text { Transcriptions }\end{array}$ \\
\hline
\end{tabular}

\section{BBC World Service}

\begin{tabular}{|c|c|c|}
\hline $\begin{array}{l}\text { Arts in } \\
\text { Action }\end{array}$ & $\begin{array}{l}\text { <http://www.bbc.co.uk/worldservice/programmes/arts.shtml> } \\
\text { arts }\end{array}$ & $\begin{array}{l}\text { Écoute } \\
\text { différée }\end{array}$ \\
\hline Discovery & $\begin{array}{l}\text { <http://www.bbc.co.uk/worldservice/programmes/disco.shtml> } \\
\text { science }\end{array}$ & $\begin{array}{l}\text { Écoute } \\
\text { différée } \\
\text { Transcriptions }\end{array}$ \\
\hline Go Digital & $\begin{array}{l}\text { <http://news.bbc.co.uk/2/hi/technology/1478157.stm> } \\
\text { technology : IT }\end{array}$ & $\begin{array}{l}\text { Écoute } \\
\text { différée }\end{array}$ \\
\hline Health & $\begin{array}{l}<\text { http://www.bbc.co.uk/worldservice/sci_tech/features/health/> } \\
\text { medicine and health }\end{array}$ & Site interactif \\
\hline
\end{tabular}




\begin{tabular}{|l|l|l|}
\hline One Planet & $\begin{array}{l}\text { http://www.bbc.co.uk/worldservice/programmes/ } \\
\text { planet_sci.shtml> } \\
\text { science: environment }\end{array}$ & $\begin{array}{l}\text { Écoute } \\
\text { différée }\end{array}$ \\
\hline
\end{tabular}

\section{NOTES}

1. L'analyse conversationnelle ou analyse de conversation (Conversation Analysis) s'est développée au début des années 1980 dans le prolongement d'un courant sociolinguistique, l'ethnométhodologie, afin d'étudier le discours dans l'interaction. Sortant du cadre de la conversation ordinaire, certains analystes ont plus particulièrement étudié les contextes professionnels et institutionnels, par exemple judiciaire, médical, scolaire, scientifique ou relatif au travail en général. Selon Drew et Heritage (1992 : 22-30), la conversation professionnelle et institutionnelle se distingue des conversations ordinaires en ce qu'elle a une visée (goal-oriented) et qu'elle répond à des contraintes spécifiques dans le cadre de procédures propres à la profession qui déterminent les choix lexicaux et syntaxiques des interactants.

2. On peut par exemple citer les travaux du professeur Edward Johnson réalisés en collaboration avec les milieux professionnels concernés. Ses recherches ont permis d'élaborer des codes langagiers internationaux dans le domaine de la navigation maritime (SeaSpeak) ou aérienne (AirSpeak), de la police (PoliceSpeak), etc. (voir Johnson 2000)

3. Le discours second le plus étudié est celui de la vulgarisation scientifique. Mais plutôt qu'une distinction binaire entre discours seconds et discours premiers, l'analyse des discours scientifiques admet l'existence d'un continuum dont les extrêmes seraient, d'une part le texte de vulgarisation pour le grand public non averti, d'autre part la communication « pointue » entre professionnels très spécialisés (Thoiron, Philippe. «Texte spécialisé et non-spécialiste : un problème de stratégie de décodage ", Revue belge de philologie et d'histoire LXIX : 629).

4. En droit anglais, une company est une société de capitaux (comme la corporation américaine) alors que la corporation anglaise est une société publique et semi-publique, constituée la plupart du temps par Charte Royale.

5. <http://www.bbc.co.uk/thenandnow/history/1920s-1.shtml>.

6. Les termes educate et education, omniprésents dans le discours de la $\mathrm{BBC}$, sont difficiles à rendre en français car leur champ lexical est très large. Ainsi, le dictionnaire Collins (French/ English dictionary on CD-ROM version 1996) propose comme traduction pour education: " éducation ", « instruction », « enseignement ", « études », «formation », «culture » et «pédagogie ». Il nous semble que la conception de service public de la BBC a voulu conserver et intégrer toutes les acceptions du terme.

7. <http://www.bbc.co.uk/thenandnow/educate_home.shtml>.

8. La prononciation et l'accent des journalistes d'information britanniques sont encore largement définis par la prononciation standard ou received pronunciation (RP), et son statut de non-accent contribue, selon Crisell (1994: 99), à effacer les marques de personnalité chez le journaliste.

9. Ce code est accessible en ligne sur le site Internet de la BBC, qui le présente en ces termes : « the $B B C$ publishes this code of ethics for programme makers, called the Producers' Guidelines, with advice on upholding standards including accuracy, impartiality, fairness, taste and decency and the welfare of children. It contains a summary of BBC Editorial Values. Under the Royal Charter BBC Governors are responsible for ensuring standards are upheld ». <http://www.bbc.co.uk/info/editorial/prodgl/ index.shtml>.

10. Cette expression est empruntée à Marc Roche, journaliste au Monde («Le départ de Christopher Bland souligne le malaise de la BBC », Le Monde, 30 avril 2001). 
11. Gavyn Davies, actuellement Président du Conseil d'Administration (Board of Governors), présente les conclusions de la commission Peacock en ces termes : «The Government established the Peacock Committee in 1985 with the hope that it would recommend in favour of advertising on BBC television. For good or ill, the end seemed to beckon for Lord Reith's BBC. Then, three things happened. First, Alan Peacock wisely rejected the advertising option. (...) Second, the supporters of the BBC re-asserted themselves. Some of these, in the private broadcasting industry, were alarmed by the threat of advertising on the $\mathrm{BBC}$, and therefore rallied behind the licence fee. But more generally, the public's respect and affection for the organisation proved deep-seated. There was no evidence of particular affection for the licence fee itself (in fact, the reverse), but nor was there any great pressure for change. Even Mrs Thatcher, increasingly irate about the $\mathrm{BBC}$, never felt able to overcome this silent force. Third, and most important, the $B B C$ embarked on a process of internal reform. Efficiency was raised until it reached levels at least compatible with those in the private sector. A commercial motive, subject to strict regulation, was injected (albeit very slowly) into the organisation through $\mathrm{BBC}$ Worldwide. The $\mathrm{BBC}$ maintained its share of the broadcasting market to a much greater extent than had been expected. And, with the political winds becoming less hostile to the concept of the licence fee, the status quo has remained the status quo. So far. » (1999. Review of the Future Funding of the BBC - Chairman's Foreword - « A Better BBC for the Digital Age ", consulté le 15 mai 2002 <http://www.culture.gov.uk/pdf/foreword.pdf >.

12. Thomas, Abigail. 1999. "Regulation of Broadcasting in the Digital Age. " Department for Culture, Media and Sport, Government research fellowship <http://www.culture.gov.uk/ creative/thomastitle.htm>.

13. The Archers date de 1951et Any Questions? de 1954.

14. On peut citer la plus ancienne émission radiophonique au monde, Letter from America, une chronique d'un quart d'heure présentée par le même journaliste, Alistair Cooke, depuis 1946 jusqu'à sa retraite en février 2004. Il décéda un mois plus tard à l'âge de 95 ans. Outre l'émission, d'une facture identique à ce qu'elle était pendant la guerre, des pages interactives sur le site Internet de la $\mathrm{BBC}$ permettaient d'écouter cette lettre en ligne en différé, d'en lire la transcription et de consulter des documents audio et vidéo. <http://news.bbc.co.uk/1/hi/world/ letter_from_america/default.stm >.

15. <http://www.bbc.co.uk/digitalradio/>.

16. La seconde station de la $\mathrm{BBC}$ fut créée pendant la seconde guerre mondiale. Forces Programme diffusait de la musique légère pour les troupes et prit ensuite le nom de Light Programme, quand National Programme devint Home Service. La troisième station, Third Programme, créée en 1946, avait des ambitions culturelles plus élevées qui ne connurent jamais un grand succès. La BBC connut une traversée du désert dans les décennies suivantes qui virent l'apparition de la télévision et d'un nouveau type de stations commerciales, étrangères ou pirates, qui diffusaient de la musique et des chansons en continu. En 1967 la BBC créa sa station musicale, Radio 1, après l'interdiction des radios pirates. Light Programme devint Radio 2, Third Programme Radio 3, et Home Service Radio 4.

17. Selon les chiffres du RAJAR (Radio Joint Audience Research Ltd), organisme indépendant qui évalue l'audience des radios publiques et privées, le taux d'audience des radios publiques était de $50,3 \%$ en 1999 , de $51 \%$ en 2000 , de 52,1\% en 2001 et de 52,6 \% en 2002 <http://www.bbc.co.uk/ radio/>. Voir aussi l'article «Radio steams ahead as TV falters ¿1» de Matt Wells (The Guardian February 1, 2002).

18. En 2002, le taux d'audience de la $B B C$ Radio ( $52,6 \%$ de l'audience globale) se répartissait de la manière suivante : $8,4 \%$ pour $\mathrm{BBC}$ Radio $1,15,7 \%$ pour $\mathrm{BBC}$ Radio 2, $1,2 \%$ pour BBC Radio $\sqrt{3}$, $11,4 \%$ pour BBC Radio 4, 4,5\% pour BBC Five Live et 11,4\% pour l'ensemble des stations locales (Source: RAJAR <http://www.bbc.co.uk/info/report2002/pdf/review_radio.pdf>). Le taux de Radio 4 continuait donc à augmenter puisqu'il était de 10,6\% en 1999 et de 11,3\% en 2000 . (Clément-Guiraud $2002: 10$ ) 
19. Historiquement, la tradition de journalisme de la $B B C$ n'a pu se développer qu'avec la $2^{\mathrm{e}}$ guerre mondiale. En effet, la $\mathrm{BBC}$ avait précédemment dû se plier aux exigences de la presse (Newspaper Proprietors' Association) qui lui interdisait de diffuser un bulletin d'information avant dix-neuf heures et de commenter les événements. Cette restriction a-t-elle joué un rôle dans le développement d'émissions à vocation plus pédagogique que strictement informative?

20. Seule exception, le magazine consacré à l'agriculture, Farming Today This Week, est programmé le samedi matin à $6 \mathrm{~h} 30$.

21. Voir en annexe une liste des magazines spécialisés de Radio 4 et BBC World Service qui proposent une présentation détaillée et des services en ligne.

22. On peut citer l'émission Home Planet dans le domaine de l'environnement, Case Notes dans le domaine médical ou Gardener's Question Time dans le domaine horticole.

23. On peut donner l'exemple de Shop Talk, émission consacrée aux problèmes du travail.

24. La régularité prend souvent une forme particulière: de nombreuses émissions sont organisées sur l'année en trois séries de deux mois, qui alternent avec trois autres séries d'une émission complémentaire. Ces couples partagent parfois le même domaine, comme Nature et Animal Farm, ou Shop Talk et Nice Work, que le site qualifie de sister programmes.

25. Dans le domaine scientifique largement représenté, on peut citer trois exemples d'émissions organisées en « série limitée » diffusées en 2002. The Serendipity of Science, série de trois émissions de 30 minutes consacrée aux découvertes scientifiques pour lesquelles le hasard a joué un rôle important, était diffusée du 27 novembre au 11 décembre, le mercredi de $21 \mathrm{~h}$ à $21 \mathrm{~h} 30$. Une première série de trois émissions, diffusée du 15 au 29 août 2002 pouvait encore être écoutée sur le site Internet. The Nation's Health, série de six émissions de 30 minutes consacrée à la santé en Grande-Bretagne depuis la deuxième guerre mondiale, était diffusée du 5 septembre au 10 octobre, le jeudi de $21 \mathrm{~h}$ à $21 \mathrm{~h} 30$, et Crude Fact, série de deux émissions de 30 minutes sur le pétrole, les mercredi 12 et 19 juin, de $21 \mathrm{~h}$ à $21 \mathrm{fih} 30$.

26. $\mathrm{BBC}$ World Service propose dorénavant une émission régulière spécialisée dans ce domaine : Go Digital.

27. Le journaliste qui créa Law in Action en 1984, Joshua Rozenberg, est barrister, Marcel Berlins, qui présenta ensuite l'émission pendant quinze ans, est solicitor. En 2004, Clive Coleman, barrister, lui a succédé.

28. Les journalistes scientifiques sont aussi bien des journalistes qui se sont spécialisés dans les sciences que des scientifiques attirés par l'univers de la radio (voir Westcott cité par Vuillaume 1997 : 652).

29. La BBC Radio s'est fait une spécialité des programmes scientifiques. Les émissions scientifiques peuvent correspondre à des genres radiophoniques différents (weekly magazines, live phone-ins, discussions, documentaries, one-off series) mais sont toutes sous la respnsabilité de $B B C$ Radio Science Unit. Deborah Cohen, qui est actuellement responsable de cette structure, est physicienne de formation. <http://www.bbc.co.uk/radio4/discover/archive_ots/cohen1.shtml >. 30. La place des juristes dans Law in Action est remarquable non seulement par leur nombre, mais aussi par le traitement discursif qui leur est fait dans l'émission (Clément-Guiraud 2002). 


\section{RÉSUMÉS}

Un rapprochement entre des études du GERAS réalisées sur des magazines d'information spécialisée à la BBC Radio dans le domaine scientifique (Science in Action sur BBC World Service) et juridique (Law in Action sur Radio 4) permet de mettre en avant des caractéristiques communes : semblables par l'approche du contenu, la visée communicationnelle, les structures formelles et discursives, ces deux émissions sont fortement ancrées dans les communautés professionnelles et scientifiques. Ces caractéristiques de forme et de fonds semblent partagées par d'autres magazines de la BBC Radio spécialisés dans différents domaines professionnels ou scientifiques. Cet article émet l'hypothèse que les objectifs et les valeurs auxquelles la BBC a toujours adhéré alliés à une grande rigueur scientifique ont permis l'émergence d'un genre radiophonique de vulgarisation particulièrement pertinent pour l'anglais de spécialité.

A comparison between GERAS studies on regular radio news and current affairs programmes about science (Science in Action on BBC World Service) and law (Law in Action on BBC Radio 4) reveals common characteristics: both programmes share similar communicative purpose, intended audience, content, formal constraints, discursive structures and they both have strong links with the academic and professional communities. These characteristics seem to be shared by other programmes regularly broadcast on the BBC Radio quality speech services in various professional or academic domains. This article suggests that the purpose and values that the BBC has developed combined with a high scientific standing have created a popularization genre of particular relevance to ESP teachers and researchers.

\section{INDEX}

Keywords : BBC, English for Specific Purposes, ESP, genre, popularisation, professional discourse, radio, specific news and current affairs

Mots-clés : anglais de spécialité, BBC, discours professionnel, genre, journalisme d'information specialisé, radio, vulgarisation

\section{AUTEUR}

\section{DANIELLE CLÉMENT-GUIRAUD}

DanielleClément-Guiraud, professeur agrégé affectée à l'IUT de Perpignan, enseigne au département “Carrières Juridiques" depuis 1993. Partant de la nécessité d'une initiation au système de common law pour aborder l'anglais de spécialité juridique dans un pays de droit romano-germanique, elle s'est intéressée au discours des professionnels qui popularisent leur domaine dans les émissions scientifiques et techniques de la BBC Radio. Suite au travail pragmatique réalisé par Pierre Vuillaume sur Science in Action, elle a analysé le discours de l'émission de la BBC Radio 4 Law in Action dans un mémoire de DEA. Daniele.Clement@univperp.fr 